\title{
Editorial
}

\section{Short Range Phenomena: Modeling, Computational Aspects, and Applications}

\author{
Carlo Cattani, ${ }^{1} \mathrm{Ming}_{\mathrm{Li}}{ }^{2}$ and Cristian Toma ${ }^{3}$ \\ ${ }^{1}$ Department of Pharmaceutical Sciences (DiFarma), University of Salerno, Via Ponte Don Melillo, \\ 84084 Fisciano (SA), Italy \\ 2 Department of Electronic Science and Technology, School of Information Science and Technology, \\ East China Normal University, Shanghai 200062, China \\ ${ }^{3}$ Faculty of Applied Sciences, Politehnica University, 060042 Bucharest, Romania \\ Correspondence should be addressed to Carlo Cattani, ccattani@unisa.it \\ Received 16 September 2008; Accepted 16 September 2008 \\ Copyright (C) 2008 Carlo Cattani et al. This is an open access article distributed under the Creative \\ Commons Attribution License, which permits unrestricted use, distribution, and reproduction in \\ any medium, provided the original work is properly cited.
}

In the recent years, the mathematical formalism of impulsive systems (based on impulsive differential equations) has tried to join together the rigorous aspects from continuous systems formalism and the wide range of applications of discrete systems formalism. They were introduced to handle many evolution processes which are subject to singular shortterm perturbations. Abrupt changes must be approached with logical, mathematical, and technical aspects dealing with the final evolution of such impulsive sources, whose effects are entirely transferred to the new state of the systems. Modern aspects in physics (quantum theory) and mathematics (wavelets, fractal theory) should be expedient in modelling short range phenomena, and describing dynamics of perturbations and transitions in natural systems (advanced materials science) and advanced systems (optic, electronic, and quantum devices).

The aim of this special issue is to present recent advances of theoretical, computational, and practical aspects for modeling short range phenomena in order to reveal new fundamental aspects in science and engineering. Using mathematical tools of wavelets analysis, fractal theory, and applied mathematics (signal processing, numerical simulations, control theory) adapted for short range phenomena, significant results were obtained in the research fields of structure analysis and image recognition, wavelets analysis of localised space-time phenomena, dynamical and computational aspects of pulse measurement, sequences of pulses and time series, and mathematical and physical aspects of pulse generation.

This special issue involves 15 original papers, selected by the editors so as to present the most significant results in the previously mentioned topics. These papers are organised as follows. 
(a) Three papers on structure analysis and image recognition: "Incremental nonnegative matrix factorization for face recognition" by Wen-Sheng Chen et al., "Direct neighborhood discriminant analysis for face recognition" by Miao Cheng et al., and "Intelligent control of the complex technology process based on adaptive pattern clustering and feature map" by Cheng Wushan.

(b) Three papers on wavelets analysis of localised space-time phenomena: "Shannon wavelets theory" by Carlo Cattani "Combined Preorder and Postorder Traversal Algorithm for the Analysis of Singular Systems by Haar Wavelets" by Beom-Soo Kim et al., and "On the discrete harmonic wavelet transform" by Carlo Cattani and Aleksey Kudreyko.

(c) Three papers on sequences of pulses and time series: "Resolution of first and second order linear differential equations with periodic inputs by a computer algebra system" by Matilde Legua et al., "Detection of variations of local irregularity of traffic under DDOS flood attack" by Ming Li and Wei Zhao, and "Tool wear detection based on Duffing-Holmes oscillator" by Wanqing Song et al.

(d) Three papers on dynamical and computational aspects of pulse measurement: "Venturi wet gas flow modeling based on homogenous and separated flow theory" by Fang Lide et al., "Detection of short step pulses using practical test-functions and resonance aspects" by Alexandru Toma and Cristian Morarescu, and "On nonperturbative techniques for thermal radiation effect on natural convection past a vertical plate embedded in a saturated porous medium" by Oluwole Makinde and Raseelo J. Moitsheki.

(e) Two papers on mathematical and physical aspects of pulse generation: "Relativistic short range phenomena and space-time aspects of pulse measurements" by Ezzat Bakhoum and Cristian Toma and "Vanishing waves on closed intervals and propagating short-range phenomena" by Toma Ghiocel and Flavia Doboga.

(f) One paper on applications of short range (localised) phenomena analysis in biology: "Solving ratio-dependent predator-prey system with constant effort harvesting using homotopy perturbation method" by Abdoul Reza Ghotbi et al.

Carlo Cattani Ming $L i$

Cristian Toma 


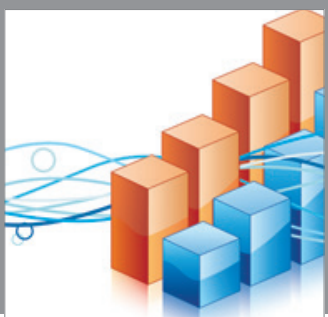

Advances in

Operations Research

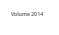

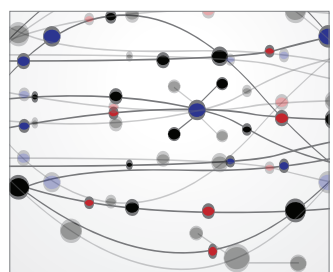

\section{The Scientific} World Journal
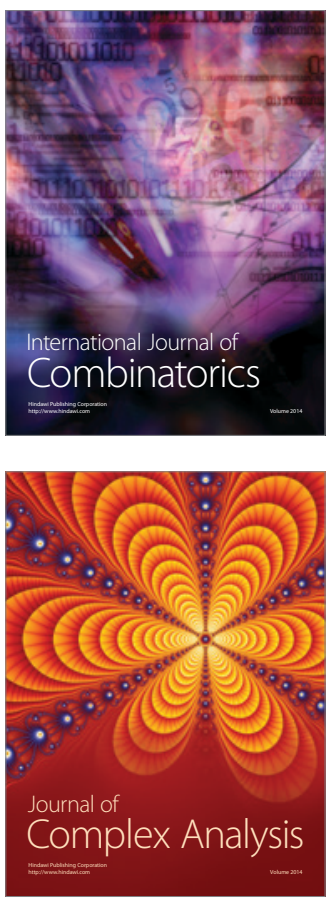

International Journal of

Mathematics and

Mathematical

Sciences
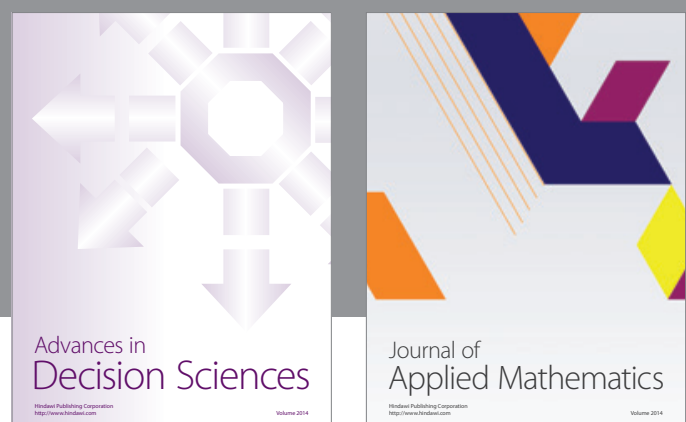

Journal of

Applied Mathematics
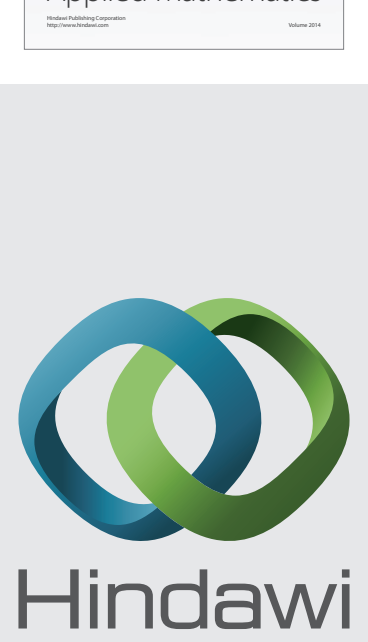

Submit your manuscripts at http://www.hindawi.com
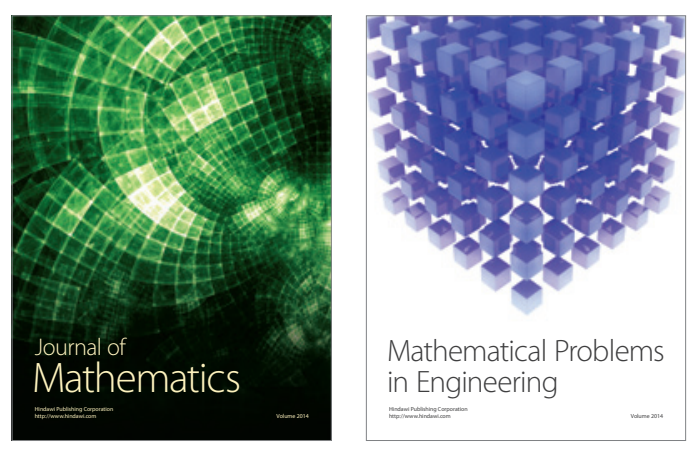

Mathematical Problems in Engineering
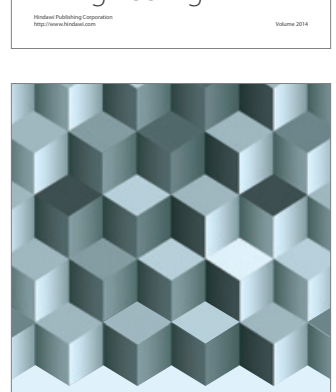

Journal of

Function Spaces
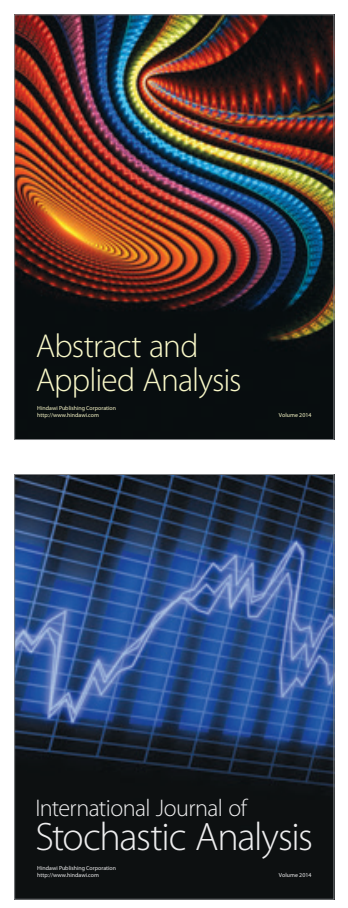

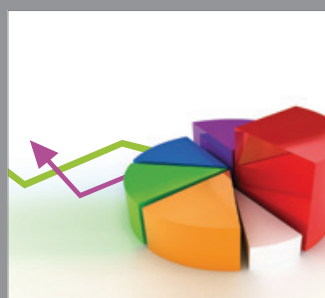

ournal of

Probability and Statistics

Promensencen
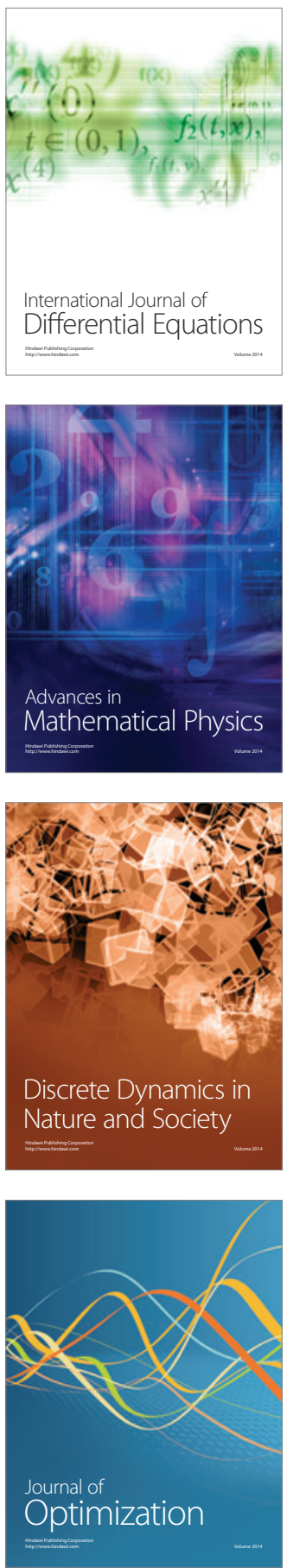